\title{
INSTRUMENTOS PARA IDENTIFICAR OS MOTIVOS PARA PRÁTICA DE ESPORTE: OPÇÕES DISPONÍVEIS NA LITERATURA
}

\author{
Bruna Hatsue Santos Yamaji \\ Universidade Norte do Paraná, Curitiba, Paraná, Brasil. \\ Dartagnan Pinto Guedes \\ Universidade Norte do Paraná, Curitiba, Paraná, Brasil.
}

\begin{abstract}
Resumo:O presente estudo de revisão procurou compilar informações disponibilizadas na literatura quanto à proposição e à validação de questionários com finalidade de identificar os motivos voltados à prática de esporte em atletas jovens. A busca de publicações foi realizada nas bases de dados Embase, Medline, PsycINFO e SPORTDiscus, considerando artigos publicados em português, inglês e espanhol. Como conclusão, vários dos questionários reunidos no estudo já foram traduzidos para o português; contudo, infelizmente, até o momento, raros foram os questionários que, em seus processos de tradução, adaptação e validação, procuraram adotar metodologia rigorosa e robusta o suficiente para atender as exigências de qualidade para uso em nosso meio.
\end{abstract}

Palavras-chave: Motivação. Treino Esportivo. Adesão. Adolescentes.

\section{Introdução}

Estudos que procuram identificar os motivos para prática de esporte e os fatores associados à adesão ou ao abandono têm constituído em temática dominante na área de conhecimento vinculada à psicologia do esporte, sobretudo em idades jovens (GILL; WILLIAMS, 2008). Também, destacam-se as diversas aproximações teóricas sugeridas para tentar explicar a conduta motivacional no contexto esportivo (DECI; RYAN, 1985; NTOUMANIS, 2001; RYAN; DECI, 2000) e o esforço direcionado à proposição e à validação de instrumentos de medida voltados à análise de selecionados motivos para a prática de esporte (BIDDLE; MARKLAND; GILBOURNE; CHATZISARANTIS; SPARKES, 2001).

O foco de análise de motivos para prática de esporte em idades jovens justifica-se com base no pressuposto de que a infância e a adolescência são períodos críticos para iniciar a participação efetiva em programas organizados de esporte, ou pelo contrário, para abandonar por completo sua prática com repercussão para idades futuras (SIRARD; PFEIFFER; PATE, 2006). Neste sentido, torna-se importante demarcar e conhecer os motivos que podem levar os jovens a praticar esporte. A identificação de motivos subjacentes ao esporte possibilita delinear ações de maneira mais efetiva que possam promover clima motivacional favorável, o que permite aos jovens maiores oportunidades de alcançarem suas metas, elevando, desse modo, as chances de aderência aos programas de esporte e, por consequência, minimizando eventual possibilidade de abandono.

Os motivos que podem mobilizar os jovens para prática de esporte são identificados, dimensionados e ordenados mediante utilização de questionários autoadministrados específicos. Via de regra, questionários para atender essa finalidade são propostos por intermédio da apresentação de itens equivalentes a determinado elenco de possíveis motivos 
previamente concebidos, agrupados em fatores de motivação, associados à pratica de esportes. Neste caso, em seu delineamento o respondente indica o grau de importância que cada item pode ter para sua prática de esporte, através de escala continua de medida do tipo Likert.

Nessa perspectiva, o campo da psicologia do esporte tem sido bastante produtivo quanto à proposição e à validação de questionários com essas características e, no momento, encontram-se disponíveis várias opções de questionários com finalidade de analisar indicadores motivacionais direcionados à prática de esporte (GILL; WILLIAMS, 2008; NTOUMANIS, 2001). Contudo, sabe-se que atributos sociais e ambientais, além do contexto cultural em que se esta inserido, deverão modular a seleção de motivos para prática de esporte. Logo, seleção adequada do questionário que melhor atende a realidade dos respondentes é fundamental para reunir informações que possam verdadeiramente repercutir o perfil motivacional dos jovens analisados. Assim, o presente estudo de revisão procurou compilar informações disponibilizadas na literatura quanto à proposição e à validação de questionários com finalidade de identificar os motivos voltados à prática de esporte em atletas jovens.

\section{Teorias de Motivação}

A motivação é tratada basicamente por um agrupamento de teorias, sendo que, mais recentemente, algumas teorias tem recebido maior atenção que outras. No entanto, de maneira unânime, as teorias de motivação recorrem fundamentalmente aos fatores de ordem intrínseca e extrínseca para tentar explicar qualquer associação com o comportamento motivado (GILL; WILLIAMS, 2008).

Neste sentido, existem várias teorias baseadas nos motivos de caráter intrínseco ou extrínseco que podem ser aplicadas na análise de motivos para prática de esporte. Contudo, mais recentemente, em razão do avanço identificado na área da psicologia, tem sido sugerido que a dicotomia intrínseca-extrínseca esta se tornando demasiadamente simplista e redutora para compreender as implicações de cunho psicológico relacionadas à motivação. Desse modo, com base em uma perspectiva autodeterminista, foi idealizada a Teoria de Autodeterminação $(\mathrm{TaD})$, mediante pressuposto de que a motivação deve ser categorizada de uma forma global, mediante continuum de posição menos autodeterminada para mais autodeterminada. Nos extremos do continuum estão localizados os constructos relacionados à desmotivação e à motivação intrínseca e, por sua vez, na parte central encontram-se os constructos identificados com a motivação extrínseca e suas respectivas regulações (RYAN; DECI, 2000). A figura 1 procura definir sucintamente as formas de motivação que constituem a TaD e os diferentes níveis de auto-regulação.

Baseando-se na $\mathrm{TaD}$, operacionalmente, a motivação intrínseca define-se em duas vertentes: (a) participação voluntária na prática de esporte, com aparente ausência de recompensas ou pressão externa; e (b) participação na prática de esporte pelo próprio interesse, satisfação e prazer que obtêm da prática. Ambos os comportamentos motivados tem por referência o divertimento, a satisfação e o prazer inerente a própria prática de esporte, idêntico ao que acontece em atividades de lazer e de ocupação do tempo livre (NTOUMANIS, 2001). Neste caso, o prazer provém unicamente da atividade em si, ao invés de recompensas extrínsecas como bens materiais, prêmios ou reconhecimento social, considerando que a participação ocorre livre de pressão e restrições. Jovens que praticam esporte por considerar interessante, por querer conhecer algo mais acerca de sua pratica e pelo prazer de superar a si próprio, são considerados como motivados intrinsecamente. Ainda, a motivação intrínseca tem origem em necessidades psicológicas de competência, autodeterminação e 
relacionamento; portanto, este constructo é fator preponderante para iniciar e permanecer na prática de esporte (BIDDLE; MARKLAND; GILBOURNE; CHATZISARANTIS; SPARKES, 2001).

\begin{tabular}{|c|c|c|c|}
\hline $\begin{array}{l}\text { Forma de Moti- } \\
\text { vação }\end{array}$ & Desmotivação & Motivação Extrínseca & $\begin{array}{l}\text { Motivação } \\
\text { Intrínseca }\end{array}$ \\
\hline
\end{tabular}

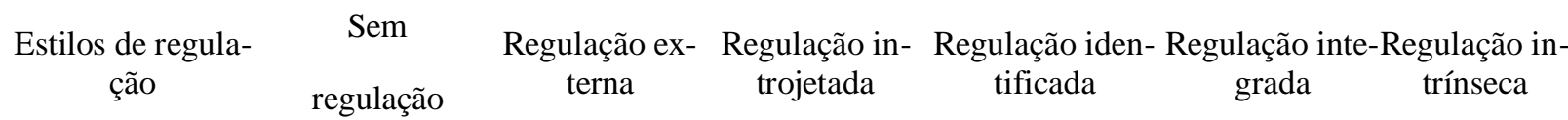

\begin{tabular}{|c|c|c|c|c|c|}
\hline $\begin{array}{l}\text { Lócus de percep- } \\
\text { ção de causalidade }\end{array}$ & Impessoal & Externo & $\begin{array}{c}\text { Parcialmente } \\
\text { externo }\end{array}$ & $\begin{array}{l}\text { Parcialmente in- } \\
\text { terno }\end{array}$ & Interno \\
\hline
\end{tabular}

Definição no cam- Pratica esporte Pratica esporte Pratica esporte Pratica esporte Pratica esporte Pratica espo do esporte de maneira pas-por obrigação para evitar pelos benefícios em razão de porte com fisiva ou não pra- ou para evitar sansões auto- que acredita pos- sua coerência nalidade em tica algum tipo de impostas sa alcançar com com outros as- si mesmo punição sua prática pectos pessoais

Figura 1 - Continuum de autodeterminação e níveis de auto-regulação aplicados a prática de esporte (adaptação de RYAN; DECI, 2000).

Quanto à definição conceitual de motivação extrínseca, verifica-se este constructo em situações em que se realiza a atividade como forma de obter alguns benefícios pretendidos, sendo que, a diversidade desses benefícios reflete a natureza multidimensional da motivação extrínseca. Assim, a motivação extrínseca consiste em conjunto variado de comportamentos, assumidos com vista a um fim claramente definido, e não pelo prazer e divertimento gerado pela própria atividade (NTOUMANIS, 2001). Logo, torna-se possível identificar diferentes estilos de auto-regulação da motivação extrínseca ao longo do continuum de autodeterminação, partindo de uma forma motivacional mais autodeterminada para a desmotivação. Ou seja, receber recompensas ou ser pressionado por algum motivo caracteriza-se por uma forma controladora de autonomia, considerando que, neste caso, este comportamento não pode ser definido como uma expressão do próprio individuo, em razão de estar subjugado a uma ação controladora. Por consequência, a sensação de autonomia ou controle reflete os extremos do continuum, assumindo a existência de diferentes tipos de motivação extrínseca de acordo com a variação do lócus de percepção de causalidade. 
Nessa perspectiva, a regulação integrada é a forma mais autodeterminada ou autônoma de determinado comportamento motivado extrinsecamente. Esta regulação é considerada como sendo volitiva, dada sua utilidade e importância para concretização de objetivos pessoais; portanto, oferecendo possibilidades de escolha. Contudo, nesta situação a ênfase não se limita exclusivamente a própria atividade, considerando que a decisão de realizar o comportamento é entendida como um esforço adicional que permite harmonizar e fornecer coerência ao que constitui a percepção que o indivíduo tem de si próprio (RYAN; DECI, 2000). Exemplo típico de caracterização da regulação integrada é o fato do jovem decidir praticar esporte por reconhecer sua importância na adoção de um estilo de vida fisicamente ativo.

A regulação identificada é caracterizada quando uma ação ou comportamento é motivado pela valorização de resultados e benefícios da atividade em questão, como é o caso, por exemplo, praticar esporte para aprimoramento da condição física e/ou para prevenção de doenças. Esta regulação é menos autodeterminada que a regulação integrada e, mesmo tendo em conta que o comportamento possa ser regulado internamente, por vezes, este comportamento é assumido sem que o indivíduo o considere agradável ou interessante, mas sim, necessário naquele momento. Desta forma, a importância é colocada no benefício da atividade (no exemplo citado, aprimoramento da condição física e prevenção de doenças) e, mesmo considerando que o comportamento é assumido sem pressão externa, representa somente um meio para alcançar o objetivo. Em síntese, a regulação identificada consiste na aceitação do comportamento por razões extrínsecas; porém, permite a percepção de algum controle e possibilidade de escolha da atividade (RYAN; DECI, 2000).

Na regulação introjetada os estilos regulatórios não são tão explícitos e torna-se mais afetivo que cognitivo. Assim, este estilo de regulação envolve a resolução de impulsos conflituosos, do tipo fazer ou não fazer, e os comportamentos resultantes provêm de reforço de pressões internas, como a culpa, o orgulho, a vergonha e a ansiedade, ou desejos de obter reconhecimento social. Neste tipo de regulação, as sanções autoimpostas são os impulsos para a ação, em oposição às limitações externas, como recompensas e punições, que dirigem a regulação. Portanto, não se verifica autodeterminação nestes comportamentos, em razão dos processos regulatórios estarem alicerçados; sobretudo, em fontes de controle externo. No contexto da psicologia, a expressão introjeção refere-se ao fato do indivíduo aceitar um princípio, mesmo não se identificando ou sem interiorizar este princípio. Exemplo da participação introjetada é a situação em que o jovem pratica esporte por acreditar que bons jovens devem praticar esporte, evitando, desse modo, o sentimento de culpa.

Reconhecida como forma de motivação não autônoma e localizada em um dos extremos do continuum de auto-regulação, a regulação externa é a forma mais básica de motivação extrínseca. Este estilo regulatório consiste na imposição de contingências externas impostas por outros indivíduos ou situação específica, sendo controlado primordialmente por recompensas, ameaças e penalizações. A regulação externa traduz o que tradicionalmente é denominado de motivação extrínseca, enquanto conceito unidimensional (NTOUMANIS, 2001). Exemplo equivalente a este estilo de motivação é o caso do jovem que pratica esporte com intenção de ter o reconhecimento do treinador ou para obter recompensa financeira ou material.

E, por fim, o comportamento desmotivado é iniciado e regulado por ações não controladas intencionalmente, consequentemente, não se considera intrínseca ou extrinsecamente motivado, tendo em vista a ausência de intenção e pensamento proativo. Desse modo, a desmotivação esta associada à relativa ausência de motivação, considerando que o 
jovem não é capaz de identificar contingências entre as ações e os resultados, não existindo, portanto, motivos para iniciar ou permanecer na prática de esporte. Se, eventualmente, o jovem vier a praticar esporte, esta será desorganizada e acompanhada de sentimento de frustração e apatia.

Os distintos estilos de regulação da motivação e as associações identificadas no continuum da autodeterminação têm sido confirmados mediante estudos de meta-análises no contexto de esporte, exercício físico e educação física (CHATZISARANTIS; HAGGER; BIDDLE; SMITH; WANG, 2003). Em síntese, assume-se que, a motivação intrínseca e determinadas formas de motivação extrínseca, como as regulações integrada e identificada, predispõe para um envolvimento psicológico mais favorável, o que favorece os indicadores motivacionais positivos. Em contrapartida, os tipos de motivação associados aos baixos níveis de autodeterminação, como as regulações introjetada e externa, correspondem a consequências motivacionais não adaptativas.

\section{Recomendações para Tradução e Validação de Questionários}

$\mathrm{Na}$ realidade brasileira, em que são bastante escassos os estudos relacionados ao campo da psicologia do esporte, tem-se duas opções para identificar os motivos que possam levar os jovens a praticar esporte: (a) idealizar e validar questionário especifico para a situação em questão; e (b) traduzir e validar para o português brasileiro questionário disponibilizado na literatura em outro idioma. Neste caso, a segunda opção parece ser a mais indicada, uma vez que evita a excessiva proliferação de questionários sobre os mesmos construtos e permite comparações mais robustas entre resultados encontrados em estudos envolvendo jovens inseridos em diferentes contextos culturais.

Contudo, traduzir e validar questionários de cunho psicológico para que sejam utilizados em outras culturas envolve mais que uma simples tradução de texto para outro idioma. Neste particular, a tradução envolve procedimentos metodológicos rigorosos, para que possa ser preservada a relevância do questionário, levando em consideração aspectos e conceitos próprios de determinada cultura, bem como, aspectos e conceitos que são universais a todas as culturas (SI; LEE, 2007).

Vallerand (1989) desenvolveu metodologia para tradução e adaptação transcultural de questionários psicológicos sistematizada em sete etapas:

5. Preparar versão preliminar, mediante recursos de tradução e retrotradução, sendo sugerido o envolvimento de dois tradutores e dois retrotradutores;

6. Analisar versão preliminar e preparar versão experimental, para comprovar se a versão retrotraduzida reflete com precisão a versão original. Para esta etapa sugere-se envolvimento de um painel de análise composto por três a cinco especialistas na área, com amplo domínio de ambos os idiomas e com experiência em tradução de textos acadêmicos;

7. Pré-testar versão experimental do questionário, em uma amostra da população a que se destina;

8. Identificar validades concorrente e de conteúdo, mediante aplicação simultânea de ambas as versões do questionário (original e traduzida) em sujeitos bilíngues da população alvo. Validade de conteúdo é tratada pelo painel de análise acionado no item anterior;

9. Identificar fidedignidade de respostas dos itens, mediante réplica de aplicação do questionário traduzido com intervalo de 2-4 semanas; 
10. Identificar validade de construto, para verificar se os itens do questionário traduzido possam medir na nova versão o construto teórico que supostamente foi idealizado para medir; e

11. Estabelecer normas de aplicação e interpretação dos resultados, para que possam ser realizadas comparações com referências apropriadas.

De destacar no campo estatístico a importância dos questionários traduzidos atenderem critérios psicométricos próprios deste tipo de medida. Neste sentido, duas importantes propriedades métricas devem ser consideradas: fidedignidade e validade de construto. No que se refere à fidedignidade, torna-se necessário considerar dois indicadores principais: (a) grau de estabilidade temporal ou reprodutibilidade, identificado mediante análise teste-reteste das respostas de cada item e das subescalas do questionário, baseada em sua aplicação ao mesmo sujeito em dois momentos distintos; porém, em condições semelhantes; e (b) consistência interna, identificada mediante análise da extensão com que um conjunto de itens contribui para definição da mesma subescala.

O grau de estabilidade temporal ou reprodutibilidade é calculado por intermédio dos coeficientes de concordância kappa ou correlação intra-classe, assumindo que, quanto mais elevado o coeficiente encontrado, maior é a clareza com que os itens são apresentados e mais estáveis no tempo são suas respostas. Valores referidos na literatura apontam como mínimo aceitável coeficiente equivalente a 0,70 , embora em alguns casos valores iguais ou superiores a 0,60 possam ser satisfatórios. Por outro lado, para o cálculo da consistência interna recorrese à estimativa do alfa $(\alpha)$ de Cronbach; assumindo que $\alpha=1$ refere-se à consistência interna perfeita. No entanto, valores de $\alpha$ excessivamente elevados podem indicar eventual redundância entre os itens do questionário. Seja como for, via de regra, tem-se adotado como referência os seguintes intervalos de valores: inaceitável $\alpha<0,60$; fraca $\alpha=0,60-0,69$; razoável $\alpha=0,70-0,79$; boa $\alpha=0,80-0,89$; excelente $\alpha \geq 0,90$ (VALLERAND, 1989).

Através da validade de constructo procura-se garantir que o questionário possa efetivamente oferecer indicações do atributo psicológico em questão, mediante verificação da estrutura de seus itens via análises fatoriais exploratória (AFE) e confirmatória (AFC). A AFE torna possível que um conjunto de itens seja reunido em fatores/subescalas específicas, ou seja, ao explorar as correlações entre os itens, permite o seu agrupamento em dimensões, estimando a quantidade de fatores/subescalas que são necessários para explicar a variância dos itens e as relações estruturais que os unem entre si. Por vezes, o processo de agrupamentos dos itens em fatores/subescala pode sugerir redução na quantidade de itens para compor a nova versão do questionário traduzido.

Pressupostos estatísticos apontam que deva existir uma correlação elevada entre as variáveis do modelo fatorial para que a AFE tenha utilidade na estimativa de fatores/subescalas comuns, sendo a medida de adequação da amostragem de Kaiser-Meyer-Olkin e o teste de esfericidade de Barlett os indicadores mais utilizados para aferir a qualidade das correlações para prosseguir, ou não, com a AFE. Ainda, os métodos mais utilizados para extração dos fatores/subescalas são análise de fatores comuns (CFA: Common Factor Analysis) e análise de componentes principais (PCA: Principal Components Analysis). Para que se possa alcançar uma solução fatorial mais clara e objetiva e que venha a maximizar os pesos fatoriais dos itens, faz-se necessário recorrer aos procedimentos de rotação dos fatores, mediante método de rotação oblíquo ou ortogonal. A decisão por utilizar um ou outro método de rotação deve ocorrer em função da correlação esperada entre os fatores/subescalas. Se, em tese, é esperado que os fatores/subescalas não estejam correlacionados entre si, sugere-se 
utilizar o método de rotação ortogonal. Porém, se é esperado que os fatores/subescalas possam se correlacionar entre si, a opção deverá recair sobre o método de rotação oblíqua. No primeiro caso, a rotação mais utilizada é a Varimax, enquanto no segundo caso é a Promax.

Após definição dos métodos a serem utilizados para realização da AFE, colocam-se em questão os critérios para determinação dos fatores/subescalas e retenção/eliminação dos itens do questionário em questão. Para tanto, no momento da decisão, sugere-se que sejam levados em consideração a seguinte combinação de critérios:

(a) Critério de Kaiser (medida da variância explicada definida na mesma métrica dos itens): reter fatores/subescalas com valor próprio igual ou superior a uma unidade (Eigenvalue $\geq 1$ ). Também, apesar de sua natureza subjetiva, deve-se analisar o gráfico do "cotovelo" (scree plot) e observar a quantidade de fatores/subescalas acima da "dobra do cotovelo";

(b) Comunalidades (proporção da variância de cada item que é explicada pelo conjunto de fatores/subescalas extraídos): valores acima de 0,50 indicam que boa parte da variância dos resultados de cada item é explicada pela solução fatorial; porém, a eliminação do item deve somente ser considerada com valores abaixo de 0,40 ;

(c) Pesos fatoriais (correlação entre item e fator/subescalas): são considerados significativos quando o valor é igual ou superior a 0,50; contudo, pode-se assumir que valores até 0,30 venham a ser relevantes; porém, deve ser considerado como mínimo para que possa ser interpretado. Pesos fatoriais superiores a 0,70 são considerados indicativos de uma estrutura muito bem definida;

(d) Pesos fatoriais cruzados: inexistência de itens com pesos fatoriais acima de 0,30 em mais que um fator. Se isso acontecer e se a diferença entre os pesos for igual ou inferior a 0,15 , deve-se considerar a eliminação do item;

(e) Proporção de variância explicada pelos fatores: devem-se reter fatores/subescalas com pelo menos $40 \%$ de capacidade explicativa. Soluções fatoriais que explicam $60 \%$ da variância dos dados são consideradas bastante satisfatórias;

(f) Consistência interna do fator: deve-se garantir valores associados ao $\alpha$ de Cronbach $\geq$ 0,70. Ainda, sugere-se analisar dois outros aspectos adicionais: o valor da consistência interna em caso de eliminação de algum item, sendo necessário que o valor de $\alpha$ não aumente caso isso aconteça, e as correlações entre o item e o valor do fator/subescala, sendo aconselhável valores superiores a 0,50 ;

(g) Retenção de fatores/subescalas com pelo menos três itens: esta regra é de extrema importância por questões de estimativa do modelo em fase posterior de validação do questionário. Contudo, muitos itens por fator/subescala também não é necessariamente a melhor opção, em razão do risco de dificultar uma verdadeira unidimensionalidade do fator/subescala.

Ainda, a quantidade de sujeitos necessária para processar uma AFE é outra preocupação que deve ser levada em conta. Neste caso, a razão de 10:1 (quantidade de sujeitos por cada item do questionário) é uma proposta de consenso na literatura (HAIR; BLACK; BABIN; ANDERSON; TATHAM, 2006).

Se, por um lado, a AFE é utilizada para explorar as correlações entre as variáveis disponíveis e identificar possíveis fatores/subescalas que explicam sua variância, por outro, a AFC é empregada para confirmar se a estrutura do modelo se ajusta adequadamente aos dados. Ao contrário do que ocorre na AFE, em que supostamente não existem informações à 
priori sobre a quantidade de fatores/subescalas e sua relação com os itens, na AFC o modelo (quantidade de fatores/subescalas, itens correspondentes e erros de medida) é definido e especificado de antemão. Em síntese, tanto a AFE como a AFC tem como objetivo reproduzir as relações observadas entre o grupo de itens e os fatores/subescalas. No entanto, diferença fundamental reside na quantidade e na natureza das especificações/restrições realizadas à priori. Para tanto, a AFC requer consistentes fundamentos empíricos e conceituais para guiar as especificações a serem estimadas no modelo.

O método mais frequentemente utilizado na AFC é o da máxima verosimilhança (Maximum Likeliood), tendo como principal objetivo encontrar as estimativas dos parâmetros como se fosse a verdadeira população, maximizando a verosimilhança da matriz de covariância dos dados com a matriz de covariância restrita pelo modelo. Neste caso, mediante teste de qui-quadrado $\left(\chi^{2}\right)$, são analisadas eventuais discrepâncias entre as duas matrizes. No entanto, analisar a adequação do modelo somente com base no teste de $\chi^{2}$ pode não ser a melhor abordagem, considerando que existem outros índices que fornecem informações bastante úteis na determinação do seu ajuste. Apesar da multiplicidade de índices para análise dos modelos não reunir consenso na literatura, parece existir tendência sustentada para utilizar os seguintes índices de ajuste:

Teste de Qui-Quadrado $\left(\chi^{2}\right)$ : oferece indicação quanto às eventuais discrepâncias entre a matriz de covariâncias dos dados e a matriz de covariância do modelo. Valores de $p$ não significativos sugerem bom ajuste;

Qui-Quadrado Normalizado $\left(\chi^{2} / g l\right)$ : corresponde ao valor de $\chi^{2}$ dividido pelo grau de liberdade (gl). Reduz a sensibilidade do teste ao tamanho da amostra e à complexidade do modelo. Valores de $\chi^{2} / \mathrm{gl}<3$ sugerem bom ajuste;

Standardized Root Mean Square Residual (SRMSR): representa o valor da média residual que deriva dos valores de ajuste entre as matrizes de correlações do modelo e dos dados observados. Valores de SRMSR $\leq 0,08$ sugerem bom ajuste;

Root Mean Square Error of Approximation (RMSEA): expressa o grau de erro do modelo, indicando a extensão com que os dados são ajustados ao modelo perfeito. Valores de RMSEA $\leq 0,06$ sugerem bom ajuste;

Comparative Fit Index (CFI): deriva da comparação das covariações do modelo hipotético com um modelo base nulo, ou seja, apresenta uma estimativa da melhoria de ajuste do modelo especificado sobre um modelo nulo em que as variáveis não estão correlacionadas. Valores de $\mathrm{CFI} \geq 0,95$ sugerem bom ajuste;

Non-Normed Fit Index (NNFI): bastante semelhante ao CFI, o que sugere utilizar apenas um deles. No entanto, o NNFI considera os graus de liberdade, incluindo, portanto, uma função de penalização para os parâmetros livres que não melhoram o ajuste. Apesar de conceitualmente similares ambos os índices oferecem diferentes correções em função do tamanho da amostra (CFI) e da complexidade do modelo (NNFI). Valores de NNFI $\geq$ 0,95 sugerem bom ajuste.

Os valores de corte recomendados como indicadores de bom ajuste foram propostos por Hu e Bentler (1999). Neste sentido, parece não existir dúvida de que esses valores de 
corte apresentam sustentação empírica bastante consistente; porém, não deve ser interpretada como regra universal, considerando que se pode correr o risco de estar rejeitando bons modelos. Portanto, deve-se ter em mente que as sugestões de valores de corte dos índices de ajuste são apenas linhas orientadoras gerais e não, necessariamente, regras definitivas.

O objetivo principal da AFC é fornecer respostas sobre o ajuste do modelo aos dados, apontando se o modelo alcançado é ou não válido. Para tanto, pressupostos estatísticos subjacentes a AFC solicitam amostras de maior tamanho que na AFE. Mesmo sendo sugerido razão de 10:1, no caso da AFC a quantidade de sujeitos é relativo a cada parâmetro a ser estimado no modelo, e não para cada item do questionário, como é proposto para a AFE.

\section{Questionários para Identificar os Motivos para Prática de Esporte}

O rastreamento dos questionários disponibilizados na literatura incluiu pesquisa nas bases de dados eletrônicas Embase, Medline, PsycINFO e SPORTDiscus, no período entre janeiro de 1980 e dezembro de 2013, independente do país de publicação. A estratégia de busca baseou-se nos descritores "motivo", "motivação", "questionário", "psicometria" e "validade", combinados com "esporte" e "treino", com os limites da pesquisa "criança", "adolescente" e "jovem", e suas traduções para os idiomas inglês e espanhol. As referências bibliográficas localizadas foram também rastreadas para localizar outras intervenções de potencial interesse e que preenchiam os critérios de seleção para o estudo.

A seleção dos artigos acompanhou procedimento proposto para levantamento de informações disponibilizadas na literatura (HIGGINS; GREEN, 2008) e baseou-se na conformidade dos limites dos descritores, tendo sido desconsiderados aqueles que, apesar de aparecerem no resultado da busca, não abordavam o tema sob o ponto de vista de proposição, validação, tradução ou adaptação de questionários para prática de esporte em idades jovens.

No levantamento bibliográfico inicial foram encontradas por volta de 500 publicações. Excluídas as publicações cruzadas redundantes, constantes em mais de uma base de dados, em um segundo momento foram consideradas 212 publicações, das quais, após atendimento de uma sequência de critérios de seleção, 10 foram consideradas para estudo. Os critérios de seleção considerados foram: (a) estudos publicados em português, inglês ou espanhol; (b) artigos originais; (c) metodologia da proposição do questionário claramente definida; e (d) indicações de validade do questionário em atletas jovens. A análise e a seleção dos artigos foram realizadas por dois investigadores sob a supervisão de um terceiro investigador, que reanalisou as publicações excluídas. Eventuais discrepâncias relacionadas às exclusões foram solucionadas por consenso dos três investigadores.

Na tabela 1 são apresentadas as opções de questionários localizadas na literatura para identificar os motivos para prática de esporte em jovens, sendo na sua maioria, concebidos para atender a população jovem de países anglo-saxões. Por esse motivo, de imediato, assume-se que, além da tradução linguística, o questionário requer a adaptação cultural do idioma utilizado na elaboração dos itens e dos conceitos subjacentes aos fatores/subescalas considerados quanto aos aspectos de motivação. Também, identificar as propriedades psicométricas do questionário traduzido e adaptado para aplicação em uma população específica torna-se de fundamental importância para garantir a qualidade das informações. 
Tabela 1 - Questionários disponibilizados na literatura para identificar os motivos para prática de esporte em jovens.

\begin{tabular}{|c|c|}
\hline Questionários & Proponentes \\
\hline Intrinsic Motivation Inventory - IMI & Ryan (1982) \\
\hline Participation Motivation Questionnaire - PMQ & Gill, Gross, Huddleston (1983) \\
\hline Sport Orientation Questionnaire - SOQ & Gill, Deeter (1988) \\
\hline $\begin{array}{l}\text { Task and Ego Orientation in Sport Questionnaire - } \\
\text { TEOSQ }\end{array}$ & Duda, Nicholls (1992) \\
\hline $\begin{array}{l}\text { Perceived Motivational Climate in Sport Questionnaire } \\
\text { - PMCSQ }\end{array}$ & Seifriz, Duda, Chi (1992) \\
\hline Sport Motivation Scale - SMS & $\begin{array}{l}\text { Pelletier, Tuson, Fortier, Vallerand, } \\
\text { Brière, Blais (1995) }\end{array}$ \\
\hline Perception of Success Questionnaire - POSQ & Robert, Treasure, Balague (1998) \\
\hline Situational Motivation Scale - SIMS & Guay, Vallerand, Blanchard (2000) \\
\hline $\begin{array}{l}\text { Inventário de Motivação à Pratica Regular de Ativida- } \\
\text { de Física ou Esportiva - IMPRAFE }\end{array}$ & Balbinotti (2004) \\
\hline Behavioral Regulation in Sport Questionnaire - BRSQ & Lonsdale, Hogde, Rose (2008) \\
\hline
\end{tabular}

Intrinsic Motivation Inventory (IMI)

O questionário mais utilizado para identificar a motivação intrínseca é o IMI, idealizado inicialmente por Ryan (1982) e posteriormente aperfeiçoado por McAuley e colaboradores (McAULEY; DUCAN; TAMMEM, 1989; McAULEY; WRAITH; DUNCAN, 1991). Originalmente o IMI apresentava duas versões: uma com 18 itens e outra com 16 itens. 
$\mathrm{Na}$ sequência, ambas as versões originais foram unidas, com eliminação dos itens redundantes, o que resultou em versão única com 23 itens, acompanhados de escala de medida de tipo Likert de sete pontos $(1=$ "Discordo Totalmente" e $7=$ "Concordo Totalmente"), repercutindo favoravelmente no aprimoramento de suas características psicométricas (McAULEY; DUCAN; TAMMEM, 1989; McAULEY; WRAITH; DUNCAN, 1991).

O IMI é um questionário que permite identificar a intensidade da motivação intrínseca e auto-regulações em relação à qualquer tipo de atividade do comportamento humano. A forma com que os itens são formulados permite substituir a expressão mais genérica de atividade praticada pela designação da atividade efetivamente praticada pelo jovem inquirido, por exemplo, prática de esporte, prática de exercício físico, aula de educação física, etc.

Informações reunidas mediante a aplicação do IMI permite constituir quatro fatores/subescalas de motivação: (a) interesse/envolvimento; (b) percepção de competência; (c) esforço/importância; e (d) pressão/tensão. Enquanto as três primeiras subescalas se referem às facetas positivas, a quarta subescala reflete faceta negativa da motivação intrínseca. Ainda, é licito considerar o somatório da pontuação dos 23 itens como indicador da motivação intrínseca global. Neste caso, as consistências internas dos fatores/subescalas, calculadas mediante valores de $\alpha$ de Cronbach, oscilam entre 0,68 e 0,85 .

De destacar que existem na literatura internacional versões do IMI traduzidas e adaptadas para vários idiomas, inclusive português europeu (FONSECA; PAULA-BRITO, 2001a). Especificamente na versão disponibilizada em português europeu, os procedimentos de validação apontaram estrutura fatorial envolvendo somente 18 itens; porém, com valores equivalentes à consistência interna discretamente inferiores aos observados na versão original.

\section{Participation Motivation Questionnaire (PMQ)}

O PMQ foi idealizado por Gill, Gross, Huddleston (1983) e caracteriza-se como um dos questionários de maior destaque na literatura. Para sua proposição os autores, baseando-se na literatura da época, elaboraram grande quantidade de itens relacionados aos possíveis motivos para prática de esporte e aplicaram experimentalmente em amostra de jovens como projeto piloto. Diante das primeiras respostas elaboraram nova versão do questionário com 37 itens, similar a versão atual existente. Na sequência, após alguns ajustes e adaptações, chegouse ao questionário definitivo, com 30 itens equivalentes ao elenco de possíveis motivos que possam levar jovens a praticar esporte, agrupados em oito fatores/subescalas de motivação: (a) reconhecimento social; (b) atividade de grupo; (c) aptidão física; (d) emoção; (e) competição; (f) competência técnica; (g) afiliação; e (h) diversão. Em seu delineamento o jovem inquirido indica o grau de importância que mais se aplica para a sua pratica de esporte, mediante escala de medida de tipo Likert de três pontos.

O questionário procura identificar a motivação dos jovens concentrando-se no ambiente esportivo de forma geral, ou seja, competitivo ou não competitivo, buscando identificar os prováveis motivos de permanência e abandono da prática de esporte. O PMQ é um dos questionários mais utilizados nos EUA, encontrando-se também, versões traduzidas e adaptadas em vários outros países. Especificamente no idioma português são disponibilizadas quatro versões traduzidas e adaptadas do PMQ. O denominado Questionário de Motivação de Actividades Esportivas - QMAD em português europeu (SERPA, 1992), Inventário de Motivação para a Prática Desportiva - IMPD (GAYA; CARDOSO, 1998) e a Escala de Motivos para Prática Esportiva - EMPE (BARROSO, 2007) em português brasileiro. 
Recentemente nova tradução e adaptação foram realizadas envolvendo jovens atletas brasileiros; porém, seus idealizados optaram por manter a denominação original de PMQ (GUEDES; SILVÉRIO NETTO, 2013).

O QMAD é uma adaptação portuguesa do PMQ que manteve na versão traduzida os 30 itens propostos inicialmente; porém, reagrupados em sete fatores/subescalas de motivação e mediante disposição acentuadamente diferente da versão original. Na sequência, Fonseca (1999) categorizou o QMAD em oito componentes: status social, forma física, competição, afiliação geral, competência técnica, afiliação técnica/equipe, emoções e prazer/ocupação do tempo livre. Porém, estudos posteriores apontaram limitações metodológicas na definição do QMAD que podem comprometer sua aplicação (FONSECA; MAIA, 2001).

O IMPD também é uma versão adaptada do PMQ e utilizou como referência a versão já traduzida para o idioma português europeu do QMAD. Para tanto, em estudo-piloto por intermédio de abordagem exploratória envolvendo jovens de 7 a 14 anos de idade, jovens foram convidados a descrever os cinco principais motivos que os levavam a praticar esporte, $o$ que reduziu arbitrariamente a quantidade de itens de 30 para 19. Neste caso, foram excluídas questões originais e inseridas novas questões. O IMPD abriga as questões em três fatores/subescalas de motivação: (a) competência esportiva; (b) amizade/lazer; e (c) saúde. Mediante análise mais detalhada identifica-se que quantidade significativa de itens inseridos no IMPD não esta presente na versão original do PMQ e seus conteúdos são claramente voltados para crianças em idades bastante precoces. Logo, parece mais lógico assumir que o IMPD não seja uma tradução/adaptação do PMQ, mas sim, instrumento inédito direcionado a identificar os motivos que levam crianças ainda não envolvidas em programas sistematizados de treino a praticar esporte.

O EMPE é uma versão traduzida e adaptada do PMQ com objetivo de ser aplicado em jovens adultos ( $\geq 18$ anos), e contem os 30 itens da versão original do PMQ com inclusão de três outros itens específicos relacionados à saúde: item 11 - "Eu quero manter a saúde"; item 32 - "Eu quero melhorar ainda mais minha saúde"; e item 22 - "Eu quero adquirir hábitos saudáveis". O EMPE abriga sete fatores/subescalas motivacionais: (a) status social; (b) condicionamento físico; (c) energia; (d) contexto; (e) técnica; (f) afiliação; e (g) saúde. Em comparação com a versão original existem duas dimensões da escala de pontuação. A primeira é com escala de medida de tipo Likert com cinco pontos $(1=$ "Nada Importante" a 5 $=$ "Totalmente Importante"). A outra com escala de medida de 11 pontos ( $0=$ "Nada Importante" a 10 = "Totalmente Importante").

A quarta tentativa de tradução do PMQ para o idioma português foi validada transculturalmente por Guedes e Silvério Netto (2013), com discretas adaptações para a população alvo, envolvendo 30 itens reunidos em oito fatores/subescalas de motivação, precedidos pelo enunciado "Eu pratico esporte para ...". Em comparação com a versão original, a versão traduzida do PMQ apresenta diferenças quanto às dimensões da escala de pontuação e ao perfil esportivo dos sujeitos selecionados em um e outro estudo. Na versão original utilizou-se escala de medida de tipo Likert de três pontos, enquanto na versão traduzida foi considerada escala de medida de cinco pontos; logo, com maior capacidade discriminatória em suas respostas. No que se refere ao perfil esportivo das amostras selecionadas em um e outro estudo, originalmente o PMQ foi aplicado em jovens engajados em programas de férias de verão envolvendo esporte em um contexto de lazer, enquanto no estudo de validação do PMQ para o idioma português os jovens se encontravam em um contexto de elevado nível de competição, participando da etapa final da principal competição juvenil do Estado do Paraná. Seus indicadores de validação estatística apontaram que, em 
conjunto, os oito fatores/subescalas de motivação podem explicar proporção de variância próxima de $67 \%$. Ainda, a solução fatorial gerada foi similar a apresentada originalmente, com consistência interna dos fatores/subescalas de motivação, apontada pelos valores de $\alpha$ de Cronbach, variando entre 0,54 e 0,83.

\section{Sport Orientation Questionnaire (SOQ)}

O SOQ foi idealizado por Gill e Deeter (1988) para identificar os motivos e as expectativas associadas à prática de esporte em atletas jovens. Neste caso, considerou-se o esporte como uma prática multidimensional relacionada às suas orientações e especificidades. A versão original do questionário foi idealizada em inglês; contudo, são disponibilizadas versões traduzidas e adaptadas em outros idiomas, o que não é o caso do idioma português. Também, existe versão do SOQ para ser aplicada em praticantes de esporte com necessidades especiais, mais especificamente em cadeirantes (SKORDILIS; KOUTSOUKI; ASONITOU; EVANS; JENSEN; KENNETH, 2001).

De maneira sintética, o SOQ procura atender basicamente três fatores/subescalas: competitividade, orientação para vitória e orientação para metas. O fator/subescala competitividade impacta consideravelmente na opção pela prática de esporte, assumindo pressupostos de que, aqueles jovens com traços mais competitivos se identificam com competição mais exacerbada e, direta ou indiretamente, buscam mais intensamente participar de competições. O fator/subescala com orientação para vitória se identifica com a importância de vencer, em que jovens orientados para vitória dimensionam seu sucesso em comparação com o desempenho de outros, e não define seus padrões pessoais. Por outro lado, o fator/subescala com orientação para metas procura identificar a importância do desempenho pessoal no esporte. Portanto, jovens orientados para metas competem consigo mesmo, o que minimiza eventuais comparações de seu desempenho com de outros.

O SOQ é composto por 25 itens, sendo 13 relacionados ao fator/subescala competitividade, seis ao fator/subescala com orientação para vitória e outros seis ao fator/subescala com orientação para metas. Os coeficientes $\alpha$ de Cronbach originalmente observados equivalem a 0,90, 0,86 e 0,80, respectivamente, o que indica elevada consistência interna para os três fatores/subescalas que compõe o questionário. Cada item é acompanhado por escala de medida de tipo Likert de cinco pontos ( 1 = "Strongly Agree" a 5 = "Strongly Disagree"). Importante ressaltar que o questionário foi projetado para analisar jovens que praticam esporte sem nenhuma restrição a experiência de prática.

\section{Task and Ego Orientation in Sport Questionnaire (TEOSQ)}

Este questionário foi idealizado por Duda e Nicholls (1992), originalmente em inglês; no entanto, é possível encontrar versões traduzidas, adaptadas e validadas para outros idiomas, inclusive para o português europeu (FONSECA; BIDDLE, 2001), com a denominação Questionário de Orientação para o Ego e para a Tarefa no Desporto (TEOSQp), e português brasileiro (HIROTA, 2006). Ainda, também é disponibilizada versão adaptada para praticantes de esporte com necessidades especiais, especificamente para cadeirantes (FLIESS-DOUER; HUTZLER; VANLANDEWIJCK, 2003).

O TEOSQ tem por objetivo analisar perspectivas de ajustes do esporte, identificando a propensão do jovem em ser orientado pela Tarefa ou pelo Ego em determinados contextos. Neste caso, os elementos direcionados para orientação voltada à Tarefa preocupam-se fundamentalmente em aprimorar as capacidades e habilidades individuais, enquanto os jovens 
cuja orientação se prende ao Ego formulam suas percepções na competência, comparando seu desempenho com o de outros praticantes. Logo, a orientação para Tarefa esta associada ao divertimento e ao desenvolvimento da sociabilidade mediante a prática de esporte; portanto, predomínio da motivação intrínseca. Por outro lado, o jovem que procura constantemente se destacar frente aos colegas e aos adversários transparece orientação para Ego e denota a maior participação da motivação extrínseca (HARDEY; GOMES; GOULD, 1996).

O questionário é constituído por 13 itens que traduzem tipos distintos de objetivos perseguidos pelos jovens com vistas ao sucesso no contexto esportivo. O respondente se posiciona frente a cada item mediante escala de medida de tipo Likert de cinco pontos $(1=$ "Discordo Totalmente" a 5 = "Concordo Totalmente", encabeçado pelo enunciado "Eu me sinto mais bem sucedido no esporte quando ...". A concepção do questionário originou estrutura de dois fatores/subescalas (Tarefa e Ego), com diversos estudos demonstrando a adequação de seus índices de consistência interna (FONSECA; PAULA-BRITO, 2005). Importante característica para preenchimento do questionário refere-se à época em que os jovens se sentem mais bem sucedidos em seu esporte, procurando se posicionar em todas os itens com base neste período.

$\mathrm{Na}$ tentativa de aprimorar as informações levantadas é oferecida versão alternativa do TEOSQ para treinadores, o que também foi traduzida, adaptada e validada para a português europeu, designado Questionário de Orientação para o Ego e para a Tarefa, versão Treinador - TEOSQpt (FONSECA; PAULA-BRITO, 2005), mas não no português brasileiro. Neste caso, a intenção é obter referência de como os jovens interpretam o modo como os treinadores configuram seus objetivos de realização. Na versão alternativa, antecedendo os 13 itens apresentados aos respondentes, distribuídos pelos dois fatores/subescalas (Ego e Tarefa), é anunciada a questão: "Para o/a treinador/a tenho mais sucesso no meu esporte quando...".

\section{Perceived Motivational Climate in Sport Questionnaire (PMCSQ)}

O PMCSQ foi idealizado por Seifriz, Duda e Chi (1992) com finalidade de analisar clima motivacional específico do esporte, como Tarefa e Ego, respectivamente intitulado de mestria e performance. Característica principal do questionário é a identificação de possíveis causas de sucesso no esporte. Os itens foram projetados para analisar grau de percepção da motivação intrínseca oferecendo ênfase ao envolvimento na mestria e na performance. Inicialmente, a partir de considerações teóricas e empíricas, o questionário foi proposto com 106 itens. Na sequência, foi reduzido para 40 itens e finalizou com 21 itens, dos quais, nove se identificam com o fator/subescala de mestria e $12 \mathrm{com}$ o fator/subescala de performance, recebendo denominação de PMCSQ-1.

Os itens do fator/subescala de mestria descrevem a ênfase no esforço/dedicação, no aperfeiçoamento das habilidades envolvidas, na percepção da contribuição de cada membro para a equipe, nos erros aceitos como parte do processo de aprendizagem e na cooperação/coesão reforçada pelos membros da equipe. Os itens do fator/subescala de performance enfatizam a rivalidade intra-equipe, o reforço baseado no alto nível de habilidade, a punição dos erros e o favorecimento aos esportistas de destaque na equipe. $\mathrm{O}$ questionário apresenta escala de medida de tipo Likert de cinco pontos ( 1 = "Discordo Totalmente" a 5 = "Concordo Totalmente") precedida pela frase "Na nossa equipe ...". Os níveis de consistência interna encontrados foram equivalentes a 0,86 e 0,77 para os fatores/subescalas mestria e performance, respectivamente. 
Mais recentemente ocorreram importantes ajustes na versão original do PMCSQ, repercutindo favoravelmente no aprimoramento de sua qualidade psicométrica, intitulada PMCSQ-2. A estrutura inicialmente proposta para o questionário foi preservada; contudo, ocorreram adaptações na formulação dos itens, estendendo para 33 itens. Neste caso, os valores equivalentes aos coeficientes de $\alpha$ de Cronbach para os fatores/subescalas equivalentes ao clima motivacional de mestria e performance se aproximaram de 0,88 (NEWTON; DUDA; YIN, 2000). Encontram-se versões traduzidas e validadas do PMCSQ-2 para outros idiomas, inclusive português brasileiro (BENCK, 2006).

Com denominação de Perceived Motivational Climate in Exercise Questionnaire (PMCEQ) e com similar estrutura de itens e subescalas foi idealizada versão direcionada especificamente para análise em situação de prática de exercício físico (THOMAS; BARRON, 2006). Adaptação realizada apresentou níveis de consistências internas equivalentes a 0,86 e 0,77 para fatores/subescala de mestria e performance, respectivamente. Contudo, não foi apresentada qualquer informação adicional sobre sua validade de construto. Versão adaptada ao exercício físico já foi traduzida para o português europeu (CID; MOUTÃO; LEITÃO; ALVES, 2012) e brasileiro (FREJOMIL; CASAL, 2009).

\section{Sport Motivation Scale (SMS)}

Respaldado pela TaD, inicialmente foi desenvolvido questionário denominado Éclelle de Motivation dans lês Sports no idioma francês (BRIÈRE; VALLERAND; BLAIS; PELLETIER, 1995). Posteriormente, quando de sua tradução e adaptação para o idioma inglês recebeu a denominação Sport Motivation Scale - SMS (PELLETIER; TUSON; FORTIER; VALLERAND; BRIÈRE; BLAIS, 1995). Mais recentemente, o SMS vem sendo traduzido para vários idiomas, sendo considerado um dos instrumentos mais utilizados para identificar os motivos para prática de esporte. A versão brasileira recebeu a denominação Escala de Motivação no Esporte - EME-BR (COSTA; ALBUQUERQUE; LOPES; NOCE; COSTA; FERREIRA; SAMULSKI, 2011).

A SMS é designada para representar especificamente o continuum da TaD e identifica diferentes formas de motivação intrínseca, motivação extrínseca e desmotivação. $\mathrm{O}$ questionário é composto por 28 itens, iniciando com o enunciado "Participo e me esforço para a prática de esporte ...", acompanhado de escala de medida de tipo Likert de sete pontos ( 1 = "Não Corresponde Nada" a 7 = "Corresponde Exatamente").

Contudo, mais recentemente, limitações teóricas e empíricas da SMS, entre outros motivos, em razão da versão original contemplar unicamente itens com conteúdo equivalente a cinco dos seis tipos de regulação motivacional previsto na $\mathrm{TaD}$, e de alguns fatores/subescalas apresentarem índices de consistência interna marginalmente inferior ao valor limite de $\alpha$ de Cronbach de 0,70, deram origem a SMS-6 (MALLET; KAWABATA; NEWCOMBE; OTERO-FERERO; JACKSON, 2007). Neste caso, os seis fatores/subescalas contemplados na SMS-6 são desmotivação, motivação extrínseca de regulação externa, motivação extrínseca de regulação introjetada, motivação extrínseca de regulação identificada, motivação extrínseca de regulação integrada e motivação intrínseca. A versão revisada SMS-6 demonstra propriedades psicométricas superiores às relatadas na versão original. 


\section{Perception of Success Questionnaire (POSQ)}

Roberts, Treasure e Balague (1998) desenvolveram questionário para identificar as orientações de metas (Tarefa e Ego) no contexto esportivo. Inicialmente, elaboraram conjunto de 48 itens selecionados a partir da literatura e de outros questionários que se dirigem à percepção de sucesso no esporte. Na sequência, mediante verificação das diferentes abordagens, o conjunto de itens foi reduzido para 26, definindo a escala inicial do POSQ. No entanto, após uma sequência de aplicações e análises estatísticas em vários estudos com objetivo de aprimorar sua qualidade, o questionário apontou validade satisfatória com não mais que 12 itens (seis itens para cada orientação de metas), definindo a versão atual do questionário.

Ainda, foram elaboradas duas outras versões do POSQ, uma especificamente para ser utilizada em adolescentes e outra em adultos. Em ambas as versões o questionário tem como finalidade a percepção de sucesso relacionado à tarefa e ao ego no esporte, sendo que os itens indagam como o respondente percebe o seu sucesso quanto à prática de esporte. Cada um dos questionários inicia com a questão "Eu me sinto realmente com mais sucesso no esporte quando ...", em que são apresentados 12 itens/situações possíveis de ocorrer no contexto esportivo, sendo que as opções de posicionamento estão dispostas em uma escala de medida de tipo Likert de cinco pontos, ancorados nos seus extremos de $1=$ "Concordo Completamente" a 5 = "Discordo Completamente".

Os índices originais de consistência interna, utilizando como referência o coeficiente $\alpha$ de Cronbach, foram de 0,92 para orientação à Tarefa e 0,90 para orientação ao Ego. Estudos traduziram e validaram o POSQ para outros idiomas atestando sua qualidade psicométrica, inclusive português europeu (FONSECA; PAULA-BRITO, 2001b) e brasileiro (BENCK, 2006).

\section{Situational Motivation Scale (SIMS)}

Elaborada inicialmente para uso no contexto educacional, a SIMS tem por objetivo analisar a motivação intrínseca e a desmotivação (GUAY; VALLERAND; BLANCHARD, 2000). Preliminarmente, a SIMS foi constituída por 26 itens; contudo, os resultados iniciais mostraram que 10 desses itens apresentavam baixa relação com itens que se identificavam no mesmo fator/subescala motivacional. Logo, foram excluídos, resultando em um instrumento composto por 16 itens.

Neste caso, o respondente se posiciona frente à questão "Why are you currently engaged in this task/activity?", mediante escala de medida de tipo Likert de sete pontos, em que 1 equivale a "Correspond not at all" e 7 "Correspond exactly", com escore intermediário de 4 equivalente a "Correspond moderately". Especificamente no contexto esportivo, a SIMS tem demonstrado satisfatórias características psicométricas, com valores equivalentes aos coeficientes de Alfa de Cronbach oscilando entre 0,63 (desmotivação) e 0,87 (motivação intrínseca). Explica-se a menor consistência interna relacionada ao fator de desmotivação em razão da menor quantidade de itens que compõe o fator/subescala (STANDAGE; TREASURE; DUDA; PRUSAK, 2003). Existem versões traduzidas e adaptadas para outros idiomas além da língua inglesa; porém, não é o caso do português europeu ou brasileiro.

Inventário de Motivação à Pratica Regular de Atividade Física ou Esportiva (IMPRAFE) 
Elaborado por Balbinotti (2004), o inventário foi idealizado com intuito de definir um instrumento de âmbito nacional, não envolvendo traduções e adaptações de instrumentos internacionais. Foi proposto para quantificar e ordenar seis possíveis fatores/subescalas associados aos motivos para prática de atividade física em um contexto amplo, inclusive envolvendo o esporte. Portanto, diferentemente dos demais questionários disponibilizados na literatura, o IMPRAFE procura identificar os motivos voltados a todo tipo de prática regular de atividade física, como é o caso de exercício físico e lazer ativo, e não especificamente a prática de esporte.

Consiste em questionário com 120 itens, agrupando 20 itens para cada um dos seis fatores/subescalas considerados: (a) controle de estresse; (b) saúde; (c) sociabilidade; (d) competitividade; (e) estética; e (d) prazer. O questionário inicia com a expressão "Realizo atividade físicalesporte para ...", em que o respondente deverá se posicionar mediante escala bidirecional, de tipo Likert, graduada em 5 pontos, transitando entre (1) "Isto me motiva pouquíssimo" e (5) "Isto me motiva muitíssimo". O questionário conta ainda com uma escala de verificação que permite analisar o nível de atenção do respondente durante sua aplicação. Seis itens, um de cada fator/subescala, tomado aleatoriamente, são repetidos no final do questionário. Logo, medida de validade da aplicação pode ser obtida mediante concordância entre as respostas.

Estudos têm confirmado a qualidade psicométrica do IMPRAFE (BALBINOTTI, 2004; BARBOSA; BALBINOTTI, 2006), apontando valores equivalentes ao coeficiente de $\alpha$ de Cronbach entre 0,89 e 0,94 para os seis fatores/subescalas de motivação considerados. No entanto, na tentativa de simplificar a aplicação do questionário e diminuir o tempo dedicado ao seu preenchimento, recursos estatísticos de análises fatoriais exploratória e confirmatória permitiram idealizar versão resumida do questionário, mantendo sua qualidade psicométrica.

A versão resumida do IMPRAFE manteve tanto a proposta original de identificar seis fatores/subescalas, quanto à estrutura de apresentação dos itens. Neste caso, o questionário é composto por 48 itens, agrupando oito itens para cada um dos seis fatores/subescalas considerados. Foi igualmente mantida a escala de verificação, de forma que juntam-se aos 48 itens, seis itens tomados aleatoriamente na própria escala. Valores equivalentes ao coeficiente de $\alpha$ de Cronbach foram identificados entre 0,82 e 0,94 para os seis fatores/subescalas de motivação consideradas (BARBOSA; BALBINOTTI, 2006).

\section{Behavioral Regulation in Sport Questionnaire (BRSQ)}

Elaborado por Lonsdale, Hogde e Rose (2008), o questionário propõe identificar os motivos para a prática de esporte em adolescentes e jovens adultos. Seus idealizadores não orientam a utilização do BRSQ em outros contextos de atividade física, como é o caso da educação física e do exercício físico, devendo, portanto, ser aplicado particularmente no meio esportivo, envolvendo atletas de competição.

Versão inicial do BRSQ é constituída por 42 itens complementares à expressão " $E u$ prático o meu esporte ....", acompanhada de escala de medida de tipo Likert de sete pontos, variando entre (1) "Não Corresponde Nada" e (7) "Corresponde Exatamente", intermediado por (4) "Corresponde Moderadamente". $\mathrm{Na}$ sequência, em análises confirmatórias de validação o questionário foi reduzido para 36 itens, reunidos em nove fatores/subescalas de regulação motivacional: (a) desmotivação; (b) motivação extrínseca de regulação externa; (c) motivação extrínseca introjetada; (d) motivação extrínseca identificada; (e) motivação extrínseca integrada; (f) motivação intrínseca para atingir objetivos; (g) motivação intrínseca 
para experiências estimulantes; (h) motivação intrínseca para conhecer; e (i) motivação intrínseca.

De maneira alternativa, é proposto o uso de medida multidimensional para a motivação intrínseca, removendo-se, desse modo, a subescala de motivação intrínseca, assumindo oito/subescalas; porém, mantendo os 36 itens, o que é denominado BRSQ-8. Ainda, medida geral de motivação intrínseca também pode ser empregada, removendo-se os fatores/subescalas de regulação motivacional associados à motivação intrínseca para atingir objetivos, à motivação intrínseca para experiências estimulantes e à motivação intrínseca para conhecer, resultando, desse modo, em análise da motivação em seis fatores/subescalas com 24 itens, o que é denominado $B R S Q-6$.

Com relação às propriedades psicométricas, em estudos envolvendo jovens de diferentes idades e experiência de prática de esporte, foram identificadas fortes cargas fatoriais, valores de consistência interna e reprodutibilidades elevados tanto para o BRSQ-8, como para o BRSQ-6 (LONSDALE; HODGE; ROSE, 2008). Ambas as versões foram traduzidas e validadas para o português brasileiro, recebendo a denominação Questionário de Regulação do Comportamento no Esporte - QRCE (VASCONCELLOS, 2011).

\section{Considerações Finais}

Independentemente do nível de exigência quanto às informações a serem tratadas, questionários autoadministrados com opções de resposta formatadas em escala de medida de tipo Likert é considerado procedimento padrão para identificar, dimensionar e ordenar os motivos relacionados à prática de esporte em idades jovens. Contudo, apesar de sua exequibilidade, os questionários com esta finalidade apresentam limitações e especificidades que, necessariamente, devem ser consideradas quando de sua utilização.

Neste sentido, encontram-se na literatura várias opções de questionários; no entanto, a quase totalidade concebida em países de origem anglo-saxão. Portanto, a aplicação desses questionários em população distinta da proposição original requer tradução e adaptação transcultural do idioma utilizado na elaboração dos itens e dos conceitos subjacentes aos fatores motivacionais considerados, além de identificação e verificação da adequabilidade das propriedades psicométricas da versão traduzida e adaptada do questionário considerado.

O uso de um mesmo questionário traduzido, adaptado e validado em vários países e culturas permite harmonizar princípios e conceitos associados aos motivos para prática de esporte especificamente aos idiomas empregados, além de facilitar eventuais comparações de seus resultados, por serem provenientes de questionários idênticos. Logo, neste caso, os protocolos de tradução e adaptação transcultural devem acompanhar procedimentos aceitos internacionalmente e a identificação das propriedades psicométricas deve atender critérios estatísticos estabelecidos previamente.

Concluindo, se por um lado, vários dos questionários reunidos na presente revisão já foram traduzidos para o português brasileiro, por outro, infelizmente, até o momento, são identificadas apenas três opções de questionários (PMQ, SMS e BRSQ) que, em seus processos de tradução, adaptação e validação, procuraram adotar metodologia rigorosa e robusta o suficiente para atender as exigências de qualidade para uso em nosso meio. 


\title{
INSTRUMENTS TO IDENTIFY SPORTS PRACTICE MOTIVES: OPTIONS AVAILABLE IN THE LITERATURE
}

\begin{abstract}
The present review study sought to compile information available in the literature regarding the proposal and the validation of questionnaires in order to identify the motives for sport participation in young athletes. The search was conducted in the Embase, Medline, PsycINFO e SportDiscus databases, considering articles published in Portuguese, English and Spanish. In conclusion, several of the questionnaires located in the study have been translated into Portuguese; however, unfortunately, yet rare were the questionnaires that in their translation, adaptation and validation processes adopted rigorous and robust methodology enough to meet the quality requirements for use in Brazil.
\end{abstract}

Keywords: Motivation. Training Sports. Adherence. Adolescents.

\section{INSTRUMENTOS PARA IDENTIFICAR LOS MOTIVOS PARA LA PRÁCTICA DEL DEPORTE: OPCIONES DISPONIBLES EN LA LITERATURA}

Resumen: Lo presente estudio de revisión intentó recopilar informaciones disponibles en la literatura acerca de la proposición y validación de cuestionarios con el fin de identificar los motivos dirigidos para la práctica del deporte en los atletas jóvenes. La búsqueda de publicaciones se lleco a cabo en las bases de dados Embase, Medline, PsycINFO y SportDiscus, considerando los artículos publicados en portugués, inglés y español. En conclusión, varios de los cuestionarios reunidos en este estudio han sido traducidos al portugués; sin embargo, desafortunadamente, todavía, son raros los cuestionarios que, en sus procesos de traducción y validación, trataron de adoptar metodología rigurosa y sólida suficiente para satisfacer los requisitos de calidad para su uso en Brasil.

Palabras clave: Motivación. Entrenamiento Deportivo. Adhesión. Adolescente.

\section{Referências}

BALBINOTTI, M. A. A. Inventário de Motivação à Prática Regular de Atividades Físicas e Esportivas (IMPRAFE-126). Núcleo de Estudos e Pesquisas em Pedagogia e Psicologia do Esporte. Porto Alegre: Universidade Federal do Rio Grande do Sul. 2004.

BARBOSA, M. L. L.; BALBINOTTI, M. A. A. Inventário de Motivação á Pratica Regular de Atividade Física (IMPRAFE-54). Laboratório de Psicologia do Esporte. Porto Alegre: Universidade Federal do Rio Grande do Sul. 2006.

BARROSO, M. L. C. Validação do Participation Motivation Questionnaire Adaptado para Determinar Motivos de Prática Esportiva de Adultos Jovens Brasileiros. Dissertação (Mestrado em Ciências do Movimento Humano). Centro Ciências da Saúde e do Esporte. Florianópolis: Universidade do Estado de Santa Catarina. 2007.

BENCK, R.T. Retreinamento das atribuições de sucesso e fracasso no esporte: uma proposta de intervenção pedagógica. Tese (Doutorado em Ciências da Saúde). Faculdade de Ciências da Saúde. Universidade de Brasília. 2006.

BIDDLE, S.; MARKLAND, D.; GILBOURNE, D.; CHATZISARANTIS, N.; SPARKES, A. Research methods in sport and exercise psychology: quantitative and qualitative issues. Journal of Sports Sciences, v. 19, p. 777-809, 2001. 
BRIÈRE, N.; VALLERAND, R. ; BLAIS, N. ; PELLETIER, L. Development et Validation d'une Mesure de Motivation Intrinséque, Extrinséque et d'Amotivation en ContexteSportif : I'echelle de motivation dam les sports. International Journal of Sport Psychology, v.26, n.4, p. 465-489, 1995.

CHATZISARANTIS, N. L.; HAGGER, M. S.; BIDDLE, S. J. H.; SMITH, B.; WANG, C. K. $\mathrm{J}$. A meta-analysis of perceived locus of causality in exercise, sport and physical education contexts. Journal of Sport and Exercise Psychology. v.25, p.284-306, 2003.

CID, L.; MOUTÃO, J.; LEITÃO, J.; ALVES, J. Tradução e validação da adaptação para o exercício do Perceived Motivational Climate Sport Questionnaire. Motriz: Rio Claro, v.18 n.4, p.708-720, 2012

COSTA, V. T.; ALBUQUERQUE, M. R.; LOPES, M. C.; NOCE, F.; COSTA, I. T.; FERREIRA, R. M.; SAMULSKI, D. M. Validação da Escala de Motivação no Esporte (SMS) no Futebol para a Língua Portuguesa Brasileira. Revista Brasileira de Educação Física e Esporte, v.25, n.3, p.537-246, 2011.

DECI, E. L.; RYAN, R. M. Intrinsic Motivation and Self-determination in Human Behavior. Nova York: Plenum. 1985.

DUDA, J.; NICHOLLS, J. Dimensions of achievement motivation in schoolwork and sport. Journal of Education Psychology. v.84, p.290-299, 1992.

FLIESS-DOUER, O.; HUTZLER, Y.; VANLANDEWIJCK, Y.C. Relation of functional physical impairment and goal perspectives of wheelchair basketball players. Perceptual Motor Skills, v. 96, n.3, p.755-8, 2003.

FONSECA, A. Atribuições em contextos de actividade física ou desportiva: perspectivas, relações e implicações. Tese (Doutorado em Ciências do Desporto). Faculdade de Ciências do Desporto e Educação Física. Porto, Portugal: Universidade de Porto. 1999.

FONSECA, A. M.; BIDDLE, S. Estudo inicial para a adaptação do TEOSQ à realidade portuguesa: questionário sobre a Orientação para a Tarefa e para o Ego no Desporto (TEOAQp). In: Fonseca, A.M. (Ed). A FCDEF-UP e a Psicologia do Desporto: Estudos sobre Motivação. Porto, Portugal: Editora da Universidade do Porto. 2001.

FONSECA, A. M.; MAIA, J. A. Analise factorial confirmatória a versão portuguesa do Participation Motivation Questionnaire. In: FONSECA, A. M. A FCDEF-UP e a Psicologia do Desporto: Estudos sobre Motivação. Porto, Portugal: Editora da Universidade do Porto. 2001.

FONSECA, A. M.; PAULA-BRITO, A. Propriedades psicométricas da versão portuguesa do Intrinsic Motivation Inventory (IMIp) em contextos de actividade física e desportiva. Análise Psicológica, v.19, n.1, p.59-76, 2001a. 
FONSECA, A. M.; PAULA-BRITO, A. Estudo exploratório e confirmatório à estrutura fatorial da versão portuguesa do Perception of Success Questionnaire (POSQ). Revista Portuguesa de Ciências do Desporto, v.1, n.3, p.61-69, 2001b.

FONSECA, A. M; PAULA-BRITO, A. A questão da adaptação transcultural de instrumentos para avaliação psicológica em contextos desportivos nacionais: o caso do Taskand Ego Orientation in Sport Questionnaire (TEOSQ). Revista Psycológica, v.39, p.95-118, 2005.

FREJOMIL, M. B.; CASAL, H. V. O clima motivacional em jovens nadadores. Educação Física em Revista, v.3, n.3, 2009.

GAYA, A.; CARDOSO, M. Os fatores motivacionais para a prática desportiva e suas relações com o sexo, idade e níveis de desempenho desportivo. Revista Perfil, v.2, n.2, p.40-51, 1998.

GILL, D.L.; GROSS, J. B.; HUDDLESTON, S. Participation Motivation in Youth Sports. International Journal of Sport Psychology, v.14, p.1-14, 1983.

GILL, D. L.; DEETER, T. E. Development of the sport orientation questionnaire. Research Quarterly for Exercise and Sport. v.59, n.3, p.191-202, 1988.

GILL D. L; WILliamS L. Psychological Dynamics of Sport and Exercise. $3^{\text {th }}$ Edition. Champaign, Illinois: Human Kinetics. 2008.

GUAY, F.; VALLERAND, R. J.; BLANCHARD, C. On the assessment of state intrinsic and extrinsic motivation: The situational motivation scale (SIMS). Motivation and Emotion, v.24, p.175-213, 2000.

GUEDES, D. P.; SILVÉRIO NETTO, J. E. Participation Motivation Questionnaire: Tradução e validação para uso em atletas-jovens brasileiros. Revista Brasileira de Educação Física e Esporte. v.27, n.1, p.137-148, 2013.

HAIR, J., BLACK, W., BABIN, B., ANDERSON, R., TATHAM, R. Multivariate Data Analysis. $6^{\text {th }}$ Edition. New Jersey: Pearson Educational, Inc. 2006.

HARDEY, L.; GOMES, G.; GOULD, D. Understanding psychological preparation for sport: theory and practice of elite-performers. London, England: John Wiley \& Sons. 1996.

Higgins J., \& Green, S. Cochrane Handbook for Systematic Reviews of Interventions. Chichester, England: John Wiley \& Sons. 2008.

HIROTA V.B. Motivação para aprendizagem esportiva no futebol de campo: um estudo com o questionário de orientação para tarefa e ego (TEOSQ). Dissertação (Mestrado em Fisioterapia). São Paulo: Faculdade de Ciências da Saúde. Universidade Metodista de Piracicaba. 2006. 
HU, L.; BENTLER, P. Cutoff criteria for fit indexes in covariance structure analysis: conventional criteria versus new alternatives. Structural Equation Modeling, v.6, n.1, p.1-55, 1999.

LONSDALE, C; HODGE, K; ROSE, E. A. The Behavioral Regulation in Sport Questionnaire (BRSQ): Instrument development and initial validity evidence. Journal of Sport and Exercise Psychology, v. 30, p.323-355, 2008.

MALLETT, C.J.; KAWABATA, M.; NEWCOMBE, P.; OTERO-FERERO, A.; JACKSON, S. A. Sports motivation Scale-6 (SMS-6): a revised six-factor sport motivation scale. Psychology of Sport and Exercise, v.8, n.5, p.600-14, 2007.

McAULEY, E.; DUNCAN, T. E.; TAMMEN, V. V. Psychometric properties of the Intrinsic Motivation Inventory in a competitive sport setting: A confirmatoryfactor analysis. Research Quarterly for Exercise and Sport. v.60, n.1, p.48-58, 1989.

McAULEY, E.; WRAITH, S.; DUNCAN, T. Self efficacy, perceptions of success, and intrinsic motivation for exercise. Journal of Applied Social Psychology. v.21, p.139-155, 1991.

NEWTON M.; DUDA J. L.; YIN Z. Examination of the psychometric properties of the perceived motivational climate in sport questionnaire-2 in a sample of female athletes. Journal of Sport Sciences. v.18, n.4, p.275-90, 2000.

NTOUMANIS N. A self-determination approach to the understanding of motivation in physical education. British Journal of Education Psychology. v.71, p.225-242, 2001.

PELLETIER, L. G.; TUSON, D. M.; FORTIER, M. S.; VALLERAND, R. J.; BRIÈRE, N. M.; BLAIS, M. R. Toward a new measure of intrinsic motivation, extrinsic motivation and amotivation in sports: the Sport Motivation Scale. Journal of Sport and Exercise Psychology. v.17, n.1, p.35-53, 1995.

ROBERTS, G. C.; TREASURE, D. C.; BALAGUÉ, G. Achievement goal in sport: the development and validation of the Perception of Success Questionnaire. Journal of Sport Sciences. v.16, p.337-347, 1998.

RYAN, R. M. Control and information in the intrapersonal sphere: An extension of cognitive evaluation theory. Journal of Personality and Social Psychology. v.43, p.450-461, 1982.

RYAN, R. M.; DECI, E. L. Self-determination theory and the facilitation of intrinsic motivation, social development and well-being. American Psychologist. v.55, p.68-78, 2000.

SEIFRIZ, J.; DUDA, J.; CHI, L. The relationship of perceived motivational climate tointrinsic motivation and beliefs about success in basketball. Journal of Sport and Exercise Psychology, v. 14, n. 4, p. 375-391, 1992. 
SERPA, S. Motivação para a prática desportiva: validação preliminar do questionário de motivação para as actividades desportivas (QMAD). In: Sobral, F.; Marques, A. (Ed.) FACDEX: Desenvolvimento somato-motor e factores de excelência desportiva na população escolar portuguesa. Lisboa: FCT. 1992. p.89-97.

SI, G.; LEE, H. Cross-cultural issues in sport psychology research. In: JOWETT, S.; LAVALlEE, D. (Eds.). Social Psychology in Sport. Champaign, Illinois: Human Kinetics. 2007. p. 279-288.

SIRARD, J. R.; PFEIFFER, K. A.; PATE, R. R. Motivational factors associated with sports program participation in middle school students. Journal of Adolescent Health. v.38, p.696703, 2006.

SKORDILIS, E. K.; KOUTSOUKI, D. ; ASONITOU, K. ; EVANS, E. ; JENSEN, B. ; KENNETH, W. Sport orientations and goal perspectives of wheelchair athletes. Adapted Physical Activity Quarterly. v.18, n.3, p.304-315, 2001.

STANDAGE, M.; TREASURE, D. C.; DUDA, J. L.; PRUSAK, K. A. Validity, reliability, and invariance of the Situational Motivation Scale (SIMS) across diverse physical activity contexts. Journal of Sport and Exercise Psychology. v.25, p.19-43, 2003.

THOMAS, J.; BARRON, K. A Test of Multiple Achievement Goal Benefits in PhysicalEducation Activities. Journal of Applied Sport Psychology. v.18, p.114-135, 2006.

VALLERAND, R. Vers une méthodologie de validation transculturelle de questionnaires psychologiques: Implications pour la recherche en langue française. Canadian Psychology, v.30, n.4, p.662-680, 1989.

VASCONCELlOS, D. I. T. Avaliação da Motivação para a Prática Esportiva em Adolescentes e Jovens Adultos Brasileiros: Validação do Questionário de Regulação do Comportamento no Esporte (QRCE). Dissertação (Mestrado em Ciências do Movimento Humano). Florianópolis: Centro de Ciências da Saúde e do Esporte, Universidade do Estado de Santa Catarina. 2011.

Recebido em:21/05/2014

Revisado em: 20/11/2014

Aprovado em: 14/01/2015

Endereço para correspondência:

bruna_yamaji@hotmail.com

Bruna Hatsue Santos Yamaji

Rua Arvelino Pelisson 504,

Bairro Jardim São Francisco,

CEP 86200-00, Ibiporã, Paraná 